\title{
Combined Auditory and Visual Cueing Provided by Eyeglasses Influence Gait Performance in Parkinson Disease Patients Submitted to Deep Brain Stimulation: a Pilot Study
}

Carolina de Oliveira Souza ${ }^{1,3}$, Mariana Callil Voos ${ }^{1,2}$, Hsin Fen Chien ${ }^{1}$, Rachael Brant ${ }^{1,3}$, Alessandra Ferreira Barbosa ${ }^{1}$ Fernanda Colucci Fonoff1, Fátima Aparecida Caromano², Luiz Carlos de Abreu4 , Egberto Reis Barbosa1, Erich Talamoni Fonoff ${ }^{1,3}$

\section{Abstract}

Background: Auditory-visual cueing using portable cueing devices has been effective for gait training in rehabilitation programs with Parkinson patients. However, it is possible that some gait problems arise due to interference from chronic high frequency stimulation with the gait and balance neural networks in PD patients. Thus, it should be useful to test whether advanced PD patients experiencing gait problems (despite the treatment with medication and high frequency deep brain stimulation - DBS) would benefit from therapy using cueing.

Methods: Eyeglasses combining auditory-visual cueing were worn by 18 patients with advanced PD and treated with medication and deep brain stimulation. Patients were assessed using the Dynamic Gait Index, Timed Up and Go and Six-Minute Walking Test and performance was measured with and without the cueing (with and without eyeglasses on).

Results: One-way ANOVAs indicated that the Dynamic Gait Index and the Six-Minute Walking Test significantly improved in the cued condition. Since cueing was task specific, and Timed Up and Go includes subtasks such sitting and standing, the combined auditoryvisual cueing did not improve performance. Conversely, the combined cueing may have worked as distractors during these subtasks.
1 Movement Disorders Clinic, Department of Neurology, Hospital das Clínicas of the University of São Paulo. School of Medicine, São Paulo, Brazil.

2 Physical Therapy, Occupational Therapy and Speech Therapy Department, University of São Paulo. School of Medicine, São Paulo, Brazil.

3 Division of Functional Neurosurgery of Hospital das Clínicas, Department of Neurology, of the University of São Paulo Medical School, São Paulo, Brazil.

4 Laboratory of studies design and cientifical writing. Departament of Public Health. Faculdade de Medicina do ABC. Santo André, Brazil.

Contact information:

Carolina de Oliveira Souza.

Address: Rua Honório Bicalho, 160 CEP 04160-120, Saúde, São Paulo, SP, Brasil.

” souzaco@ig.com.br 
Conclusion: Combined auditory-visual cueing provided by this wearable technology may have practical applicability in rehabilitation. It provided additional benefits on gait in patients with advanced PD with deep brain stimulation in the subthalamic nucleus.

\section{Keywords}

Parkinson disease; deep brain stimulation; gait.

\section{Introduction}

Gait in Parkinson disease (PD) is characterized by difficulty starting and stopping, reduced step length and walking speed, increased gait cadence, and freezing $[1,2]$. Such impairments contribute to a greater risk of falls and loss of functional independence $[1,2]$. Despite the benefits of dopaminergic medication [3] and high frequency deep brain stimulation $[3,4]$, therapeutic effectiveness on gait impairment remains limited.

Deep brain stimulation became one of the most applied treatments for advanced PD because of its efficacy and it will probably be indicated for a larger number of PD patients in the future $[3,4]$. While motor complications from PD are suitably treated by deep brain stimulation, the treatment of many gait impairments remains to be solved. Yet, it is not clear if these gait problems develop as a natural course of the disease itself or if chronic high frequency stimulation interferes with the gait and balance neural networks in PD patients $[4,5]$.

Clinical evidence supports the use of auditory and visual cues to increase stride length and regulate gait cadence in PD $[6,7]$. Visual cues, most commonly delivered by lines on the floor, direct the patient to take larger and more regular steps [7]. As visual cues are often unavailable outside the laboratory setting, devices that exploit portable and at-home sensory cueing have been developed [8].

The efficacy of combined auditory and visual cueing devices has been explored in several studies of PD patients $[8,9]$. One study evaluated patients in off-state (without medication) gait impairment at baseline and after two weeks of daily at-home use.
After the training, patients increased gait speed and stride length, even after the removal of the cueing device. The residual benefits suggest that continuing sensory information provided by the device can induce plasticity in the neural network involved in gait and could be used for rehabilitation [8].

It is unclear whether deep brain stimulation of the subthalamic nucleus would interfere with gait and balance neural networks. Besides, it is unknown whether combined auditory and visual cueing would benefit the gait of patients with advanced PD treated with deep brain stimulation.

The purpose of this study was to test the effect of simultaneous auditory and visual cueing provided by eyeglasses on advanced PD patients with gait impairment, chronically treated by dopaminergic medication and high frequency stimulation of the subthalamic nucleus. If these patients show significant improvement, then it suggests that cueing could be useful even in patients with advanced symptoms.

\section{Methods}

\section{Patients}

Eighteen PD outpatients, with mean age 66.7, SD 5.9; 13 males; UPDRS - III [10] 20.1, SD 5.4; Mini Mental State Examination score 27.9, SD 1.9 [11]; Wisconsin Sorting Cards (Errors) [12] 20.5, SD 8.9 from the Movement Disorders Clinic of the Department of Neurology and the Division of Functional Neurosurgery of Hospital das Clínicas of the School of Medicine of University of São Paulo par- 
ticipated. All of them met the inclusion criteria (see below) and were invited to participate in this pilot study. All patients signed the informed consent before enrollment. The local ethical committee approved the study.

The inclusion criteria were: diagnosis of PD (according to the clinical diagnostic criteria of the United Kingdom Parkinson's Disease Society Brain Bank [13]), Hoehn \& Yahr stage 3 or 4 [14], on dopaminergic medication in optimized daily dosage during the last four weeks, and bilateral deep brain stimulation of the subthalamic nucleus with stable parameters (Medtronic 3387 electrodes, implanted three months before or earlier the enrollment, with the guidance of MRI and microelectrode recordings to locate the second of four contacts as the active point of stimulation with frequency $>130 \mathrm{~Hz}$, pulse width $90 \mu$ s and voltage between 2 and $5 \mathrm{~V}$ ).

Patients who had other neurological, orthopedic, psychiatric diseases or visual/ hearing problems; less than four years of formal education or cognitive impairment (detected by the cut-points for each educational status, according to the Brazilian version of Mini Mental State Examination: 23 for people with 4 years of formal education, and 26 for those with 5-16 years of formal education) [11] were excluded in this study.

The inclusion criteria were: diagnosis of PD (according to the clinical diagnostic criteria of the United Kingdom Parkinson's Disease Society Brain Bank [13]), Hoehn \& Yahr stage 3 or 4 [14], on dopaminergic medication in optimized daily dosage during the last four weeks, and bilateral deep brain stimulation stimulation of the subthalamic nucleus with stable parameters (Medtronic 3387 electrodes, implanted three months before or earlier the enrollment, with the guidance of MRI and microelectrode recordings to locate the second of four contacts as the active point of stimulation with frequency $>130 \mathrm{~Hz}$, pulse

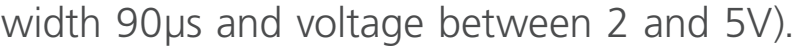

Patients who had other neurological, orthopedic, psychiatric diseases or visual/ hearing problems; less than four years of formal education or cognitive impairment (detected by the cut-points for each educational status, according to the Brazilian version of Mini Mental State Examination: 23 for people with 4 years of formal education, and 26 for those with 5-16 years of formal education) [11] were excluded in this study.

\section{Measures}

The unified Parkinson's disease rating scale (UPDRS) is used to follow the longitudinal course of Parkinson Disease. [10] It is made up of Part I (evaluation of mentation, behavior, and mood), Part II (selfevaluation of the activities of daily life, including speech, swallowing, handwriting, dressing, hygiene, falling, salivating, turning in bed, walking, and cutting food), Part III (clinician-scored monitored motor evaluation), Part IV (complications of therapy), Part $\mathrm{V}$ (Hoehn and Yahr staging of severity of Parkinson disease) and Part VI (Schwab and England scale). These are evaluated by interview and clinical observation. Some sections require multiple grades assigned to each extremity. In the present study, Parts III and $V$ were used. Part $V$ is a commonly used system for describing how the symptoms of Parkinson Disease progress. It includes stages from 1 to 5. [14]

The Dynamic Gait Index tested the ability to modify gait in response to task demands [18]: walking on a level surface, changing gait speed, walking with vertical and horizontal head turns, pivot turning, stepping over an obstacle and ascending and descending stairs. Items were scored on a 4-point ordinal scale. Zero represented severe impairment and three represented normal movement (range: 0 - 24). Dynamic Gait Index, is a good indicator of fall status for healthy elderly and patients with PD. [18]

The Six-Minute Walking Test combined numerous turns and 30-m straight-line walking within 6 minutes. It measured walking capacity and involved walking back and forth down a corridor. Previous studies showed that it was suitable for individuals with PD [17]. 
The Timed Up and Go measured basic mobility skills of healthy elderly or patients with PD. It included standing, walking for 3 meters, turning, returning to the chair and sitting. The time to complete the entire sequence is registered in seconds [16].

\section{Procedures}

Patients were assessed individually at the hospital outpatient unit. After receiving detailed explanation about the study, they signed a written informed consent approved by the Ethics Committee of the School of Medicine of University of São Paulo.

All patients were assessed in the "on-medication" and the "on-deep brain stimulation of the subthalamic nucleus" condition. They were instructed to take their medication 40 to 60 minutes before the evaluation and testing, which ensured that they were in an effectively medicated state. Gait assessment consisted on the Dynamic Gait Index [15], the Timed Up and Go test [16] and the Six-Minute Walking Test [17]. Session III of the Unified Parkinson's Disease Rating Scale (UPDRS-III) [10] were used for descriptive motor assessment.

Gait evaluation was performed by three examiners graduated in Physical Therapy and experienced with patients with movement disorders. Fifty percent of the subjects, randomly assigned, performed the tests firstly with cues and then without the cues and the other fifty performed the tests in the opposite order. The total duration of assessment was 40 minutes and consisted of the following sequence, including 2-3 minutes rests between each test whenever necessary: 1) UPDRS-III: 10 minutes; 2) Dynamic gait index: 15 minutes (eyeglasses on and off conditions); 3) Six-minute walking test: 10 minutes (eyeglasses on and off conditions) and 4) Timed up and go: 5 minutes (eyeglasses on and off conditions). The condition order (eyeglasses on and eyeglasses off) was randomized for the dynamic gait index, six-minute walking test and timed up and go test.

The device was composed of a measurementcomputation unit attached to the patient's clothing, a head-mounted microdisplay, and earphones. The measurement-computation unit was composed of a multiaxial accelerometer, a compass, and a microcontroller. The apparatus, operating in an adaptive closed-loop mode, displayed a life-size virtual checkerboard-tiled floor superimposed on the real world with specialized see-through glasses. The feedback concept implied that the speed of the cues was not externally set but, rather, an outcome of the walking speed. The visual effect was the same as that created by walking over earth-stationary cues (i.e., a real tiled floor) [8] (Figure 1).

Patients heard the auditory cues through the earphones. The rhythm of the auditory cues was determined by the rhythm of the steps. The virtual floor responded dynamically to the patient's motion and "moved" toward him/ her at the speed set by the user as measured by the accelerometer. The grid allowed for patients to step on the tiles with long strides as they walked, at the same time they could view real-world obstacles without difficulty. The concomitant use of corrective lenses was possible. The auditory cueing was continuous, producing a rhythm that was readjusted based on gait speed. For the adaptation with the apparatus, before testing, patients walked for five minutes with the eyeglasses "on" (with cues), followed by five minutes walking with the eyeglasses "off" (noncued).

\section{Analysis}

Descriptive statistics were used to characterize the age and the scores on the clinical scales (Hoehn \& Yahr, UPDRS-III, dynamic gait index, six-minute walking test and timed up and go). Scores displayed a normal distribution (tested by Kolmogorov-Smirnov tests) and one-way ANOVAs were used to compare the test conditions (eyeglasses on condition vs. eyeglasses off conditions) with alfa $<0.05$. Statistica 12.0 (StatSoft Inc., Tulsa, OK) was used to run the analyses. 
Figure 1: Eyeglasses, earphones and measurement-computation unit.

\section{Results}

The mean score on DGI was $18.1 \pm 5.4$ on eyeglasses on condition and $16.2 \pm 6.7$ on eyeglasses off condition (Figure 2A, Video 1). The ANOVA including the two conditions ("eyeglasses on": patients walking with the aid of the cues versus "eyeglasses off": patients walking without the cues) showed a significant effect (F1, 17=11.39, $p=0.003$, partial eta-squared=0.898).

There was a significant improvement on the sixminute walking test when eyeglasses on condition $(297.3 \pm 87.6 \mathrm{~m})$ and eyeglasses off condition (272.6 \pm 94.2$)$ were compared $\left(F_{1,17}=6.31, p=0.022\right.$, partial eta-squared=0.917) (Figure 2B). Timed up and go score with eyeglasses on was $11.6 \pm 9.1 \mathrm{~s}$ and $16.5 \pm 23.3$ with eyeglasses off. This difference was not significant $\left(F_{1,17}=0.47 . p=0.504\right.$, partial etasquared=0.446).

ANOVAS compared the performance of both task orders (ON - OFF vs. OFF - ON). No significant differences were found for dynamic gait in- $\operatorname{dex} F_{1,16}=0.985 ; p=0.336$ and Six-minute walking test $F_{1,16}=0.039, p=0.846$. Therefore, no carry over effect was observed during the protocol.

\section{Discussion}

This study evaluated the influence of combined auditory-visual cueing on gait performance of Parkinson Disease patients with deep brain stimulation of subtalamic nucleus. We investigated whether patients with advanced PD and deep brain stimulation of the subthalamic nucleus would still have plasticity in changing their gait, and thus, whether the cueing should be effective for these patients.

Our results showed that PD patients with deep brain stimulation of the subthalamic nucleus performed better on dynamic gait index and six-minute walking test when they received combined auditoryvisual cues provided by a wearable technology. There were no differences between cued and noncued performance on the timed up and go. Although the six-minute walking test involves mainly endu- 
Figure 2A: Performance on the Dynamic Gait Index with and without auditory and visual cues.

Figure 2B: Performance on the Six-Minute Walking Test with and without auditory and visual cues.

2 A Performance on the Dynamic Gait Index with and without auditory and visual cues $F(1,17)=11.399 ; p=0.00359$

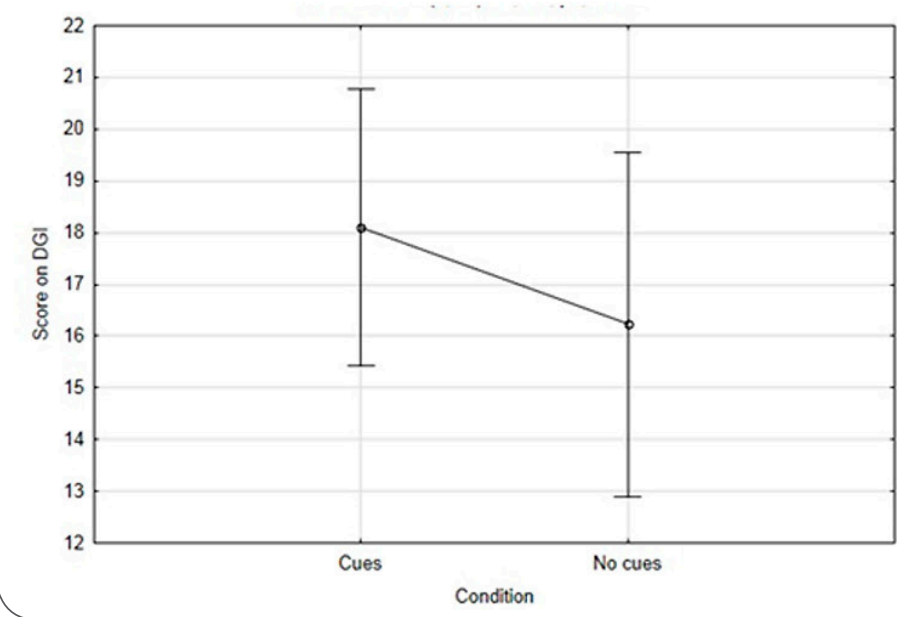

2 B Performance on the Six-Minute Walking Test with and without auditory and visual cues $F(1,17)=6.308 ; p=0.02240$

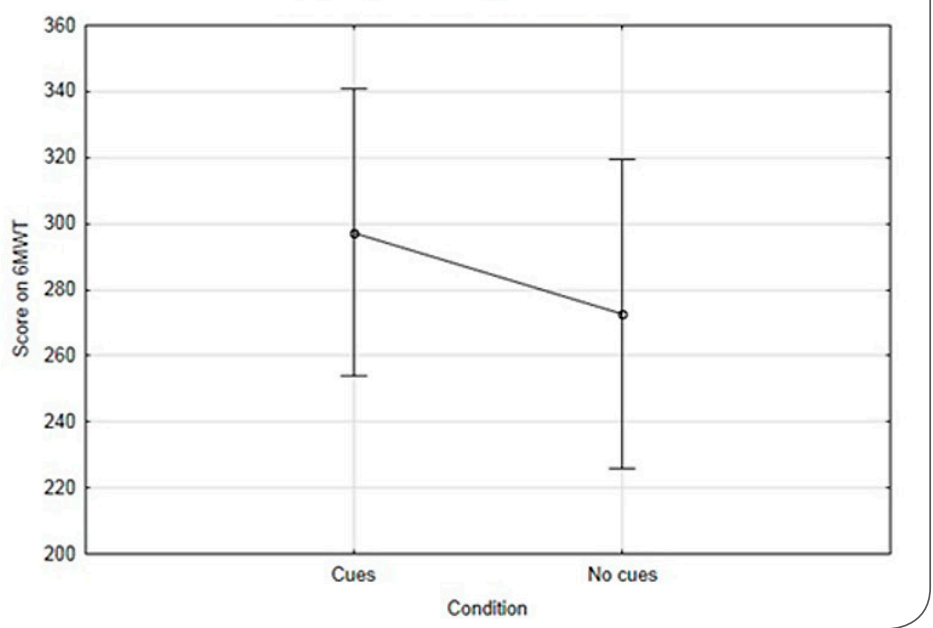

rance and walking capacity and dynamic gait index involves the functional independence on gait tasks, the performances on both tests improved on "eyeglasses on" condition. It is possible that the auditory cues helped the patients adjust the correct frequency of pacing (cadence) and the visual cues showed the proper place for stepping (step length) in the dynamic gait index and six-minute walking test.

Some factors may explain the nonsignificant effects of the cueing on the timed up and go. Firstly, the cueing given by the eyeglasses was specific for walking. It was not designed to assist sitting and rising from a chair, which are also evaluated in this test. Additionally, the patients were advanced PD patients, who usually present difficulty sitting and rising from a chair.

In a previous study, sit-to-stand improved with specific combined auditory-visual cueing training [19]. PD patients were randomly assigned to a control group or a group receiving auditory-visual cues sit-to-stand training ( 3 times per week for 4 weeks). Trained patients showed greater stability and velocity when the center-of-mass position and velocity, the backward/ forward stability at seat-off and movement termination were analyzed [19]. Thus, specific task cueing seems to be effective. However, the eyeglasses were not designed to cue for the sit-to-stand task, so they were not expected to have an effect.

Secondly, timed up and go measured the performance of multiple tasks, mostly postural transitions, and generated large confidence intervals and variability in performance. These postural transitions probably were not influenced by the cues provided by the eyeglasses. It is even possible that the combined cues may have worked as distractors in this test.

An application of the present results is that portable devices should be developed for advanced PD patients with deep brain stimulation of the subthalamic nucleus, most of whom show gait difficulties even after many therapeutic and surgical resources have been indicated. Auditory cues have been shown to help motor training in patients submitted to deep brain stimulation of the subthalamic nucleus $[8,9,20]$.

For example, Stegemöller et al. (2013) demonstrated improvement of performance by combining deep brain stimulation and cues [20]. They exami- 
ned the effects of deep brain stimulation of the subthalamic nucleus on the amplitude of repetitive finger movement across a range of external pacing rates. Nine patients with deep brain stimulation (on and off stimulation) and nine matched healthy adults performed repetitive index finger flexion movements paced by an acoustic tone at increased from 1.0 to $3.0 \mathrm{~Hz}$. On stimulation, movement rate improved in patients with bradykinesia. Deep brain stimulation improved movement amplitude across high and low rate paced cues. Although the use of cueing from a device available outside testing/ therapy environment has not been analyzed, the results suggested that patients benefit from the association between treatments (e.g. cues and deep brain stimulation) [20].

Lopes et al. 2014, utilized a smartphone that provided auditory cues, named Listenemee ${ }^{\circledR}$. Ten PD patients, five of them with deep brain stimulation of the subthalamic nucleus, with gait impairments, including freezing, underwent observation using a gait analysis laboratory. Under auditory cues the patients improved cadence, walking speed and stride length of gait [9].

Vibratory cues provided by a shoe device improved gait measures after one week of twice daily therapy. After the training, significant improvement in peak heel and toe pressure timing, and stance to swing ratio were observed. Although the results were positive, they are difficult to generalize, because the study included only two patients with PD (one with and one without STN-DBS) [21].

Combined auditory-visual cueing provided by portable eyeglasses improved gait in advanced PD with STN-DBS. This suggests practical applicability in therapy and functional gait activities and justifies further testing and customization of new wearable technologies in rehabilitation programs. Wearable technologies can be useful in health promotion [22].

As limitations, we must mention the absence of extensive training and follow-up, which would allow the assessment of a possible modification on gait performance on daily life activities. These measures might clarify if there were residual benefits and long term retention of training. The present study is useful for identifying specific hypotheses, and suggests patients with Parkinson Disease, even those at advanced stages, still have plasticity and can still benefit from gait and pace training.

As further directions, patients should wear the eyeglasses regularly and a long term follow-up should be provided to assess learning. Future studies should investigate gait parameters, with threedimensional gait analysis to characterize specific alterations of movement patterns. Another limitation was the lack of freezing analysis, which was frequently observed in our pilot study, but not systematically quantified. Future studies should include more thorough gait assessment and include specific tests, such as the freezing of gait questionnaire.

\section{Competing Interests}

The authors declare that they have no competing interests.

\section{Acknowledgements}

Carolina Souza received a grant from Movement Disorders Society to attend the 2014 Movement Disorders International Congress and presented the abstract of this work on a poster. For the remaining authors none was declared.

\section{References}

1. Nieuwboer A, Giladi N. Characterizing freezing of gait in Parkinson's disease: models of an episodic phenomenon. Mov Disord. 2013; 28: 1509-1519.

2. Dibble LE, Lange M. Predicting falls in individuals with Parkinson disease: a reconsideration of clinical balance measures. J Neurol Phys Ther 2006; 30: 60-67.

3. Pötter-Nerger $M$, Volkmann J. Deep brain stimulation for gait and postural symptoms in Parkinson's disease. Mov Disord. 2013; 28: 1609-1615 
4. Schuepbach WM, Rau J, Knudsen K, Volkmann J, Krack P, Timmermann L, Hälbig TD, Hesekamp H, et al. EARLYSTIM Study Group. Neurostimulation for Parkinson's disease with early motor complications. N Engl J Med. 2013; 368: 610-22.

5. Annic A, Moreau C, Salleron J, Devos D, Delval A, Dujardin K, Touzet G, Blond S, Destée A, Defebvre L. Predictive factors for improvement of gait by low-frequency stimulation in Parkinson's Disease. 2014 J Parkinsons Dis. 2014 Epud ahead of print.

6. Griffin HJ, Greenlaw R, Limousin P, Bhatia K, Quinn NP, Jahanshahi $M$. The effect of real and virtual visual cues on walking in Parkinson's. disease. J Neurol. 2011: 258: 991-1000.

7. Schlick C, Struppler A, Boetzel K, Plate A, Ilmberger J. Dynamic visual cueing in combination with treadmill training for gait rehabilitation in Parkinson disease. Am J Phys Med Rehabil 2012; 91: 75-79

8. Espay AJ, Baram Y, Dwivedi AK, Shukla R, Gartner M, Gaines L, Duker A, Revilla R. At-home training with closed-loop augmentedreality cueing device for improving gait in patients with Parkinson disease. J Rehabil Res Develop 2010; 47: 573-582.

9. Lopez CWO, Higuera CAE , Fonoff ET, Souza CO, Albicker U, Martinez JAE. Listenmee and Listenmee smartphone application: synchronizing walking to rhythmic auditory cues to improve gait in Parkinson's disease. Mov Sci .2014;37: 147-156.

10. Metman LV, Myre B, Verney N, Hassin-Baer S, Arzbaecher J, Sierens D, Bakay R. Test-retest reliability of UPDRS- III, dyskinesia scales, and timed motor test in patients with advanced Parkinson's disease: an argument against multiple baseline assessments. Mov Disord 2004; 19: 1079-84.

11. Brucki SMD, Nitrini R, Caramelli P, Bertolucci PHF, Okamoto IH. Sugestões para o uso do mini-exame do estado mental no Brasil. Arq Neuro-Psiquiatr 2003; 61: 777-81.

12. Slick F. Clinical normative data for the WCST-64 following uncomplicated mild head injury. Neuropsychol. 2007; 7: 247-251

13. Litvan I, Bhatia KP, Burn DJ, Goetz CG, Lang AE, McKeith I, et al, Movement Disorders Society Scientific Issues Committee. Movement Disorders Society Scientific Issues Committee report: SIC Task Force appraisal of clinical diagnostic criteria for Parkinsonian disorders. Mov Disord. 2003; 18: 467-86

14. Hoehn MM, Yahr MD. Parkinsonism: onset, progression and mortality. Neurology. 1967; 17: 427-442.

15. Tinetti ME. Performance-oriented assessment of mobility problems in elderly patients. J Am Geriatr Soc. 1986; 34: 119-126.

16. Podsiadlo D, Richardson S .The Timed "Up \& Go": a test of basic functional mobility for frail elderly persons. J Am Geriatr Soc.1991; 39: 142-148.

17. Falvo MJ, Earhart GM. Six-minute walk distance in persons with Parkinson disease: a hierarchical regression model. Arch Phys Med Rehabil. 2009; 90: 1004-1008

18. Huang $\mathrm{SL}$, Hsieh $\mathrm{CL}$, Wu RM, Tai CH, Lin CH, Lu WS. Minimal detectable change of the timed "Up \& Go" test and the dynamic gait index in people with Parkinson disease Phys Ther. 2011; 91: 114-121.
19. Bhatt T, Yang F, Mak MKY, Christina WY, Chan H, Pai YC. Effect of externally cued training on dynamic stability control during the sit-to- stand task in people with Parkinson disease. Phys Ther. 2013; 93(4): 492-503.

20. Stegemöller EL, Zadikoff C, Rosenow JM, Mackinnon CD. Deep brain stimulation improves movement amplitude but not hastening of repetitive finger movements. Neurosci Lett. 2013; 552: 135-139.

21. Winfree KN, Pretzer-Aboff I, Hilgart D, Aggarwal R, Behari M, Agrawal SK. The effect of step-synchronized vibration on patients with Parkinson's disease: case studies on patients with freezing of gait or an implanted deep brain stimulator. IEEE Transactions on Neural Systems and Rehabilitation Engineering 2013; 21: 806-811.

22. Atrash HK, Carpentier R. The evolving role of public health in the delivery of health care. Journal of Human Growth and Development. 2012; 22(3): 396-9.

\section{Comment on this article:}
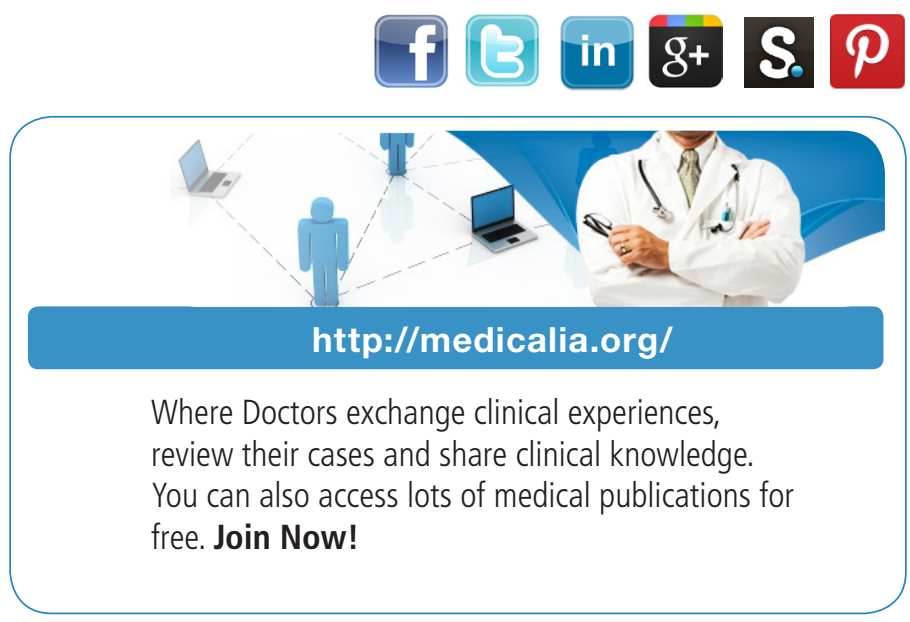

\section{Publish with iMedPub}

http://www.imed.pub

International Archives of Medicine is an open access journal publishing articles encompassing all aspects of medical science and clinical practice. IAM is considered a megajournal with independent sections on all areas of medicine. IAM is a really international journal with authors and board members from all around the world. The journal is widely indexed and classified Q1 in category Medicine. 